\title{
Preoperative oral carbohydrate drink improves surgical outcome
}

\section{Effects of preoperative fasting and surgery on metabolic state}

Elective surgeries have traditionally been performed in the overnight fasted state. ${ }^{1}$ The strict nothing by mouth rule after midnight has been in practice to decrease the volume of gastric contents at the time of anesthesia to prevent aspiration..$^{2}$ The postsurgical period is associated with breakdown of body fat and protein leading to depletion of fat and protein stores. The overnight fasting adds an additional catabolic stress to the patients. ${ }^{2}$ Both preoperative fasting and surgery cause significant decrease in insulin sensitivity. The degree of insulin resistance is directly related to the magnitude of surgery. ${ }^{3}$ Insulin sensitivity was found to be reduced by approximately $50 \%$ after major elective surgeries including abdominal and orthopedic surgeries. ${ }^{1}$ The reduction in insulin sensitivity remains for around 5 days after major surgery and it takes around 3 weeks for it to completely normalize. ${ }^{1}$ The most important factor in the development of insulin resistance has been identified to be hypo caloric nutrition. ${ }^{1}$ Decreased insulin sensitivity in peripheral tissues, mainly in skeletal muscles causes decreased glucose uptake and decreased oxidative glucose utilization. ${ }^{3}$ Decreased glucose uptake is secondary to defect in insulin stimulated translocation of glucose transporter type 4 (GLUT4). ${ }^{1}$ These changes in skeletal muscles lead to decreased carbohydrate oxidation and increased fat oxidation, which leads to preservation of glucose during fasting. The decrease in carbohydrate oxidation during fasting is caused by higher expression of Pyruvate dehydrogenase kinase (PDK), which causes inactivation of mitochondrial Pyruvate dehydrogenase complex (PDC), the enzyme that controls entry of carbohydrate derived pyruvate into Krebs cycle. The reduced anti lipolytic effect of insulin causes increase in Free Fatty Acids (FFA) which correlates with higher expression of PDK. ${ }^{3}$ The reduced inhibitory effect of insulin on protein break down causes net nitrogen loss. ${ }^{1}$ During stress, insulin clearance is enhanced as well, further complicating insulin resistance state. ${ }^{1}$ Hormones like cortisol, catecholamine and glucagon are released during stress and elective surgery and have metabolic effects opposing those of insulin. ${ }^{1}$ Reduced insulin sensitivity blunts the anabolic effects of growth hormone. Growth hormone exerts anabolic effects by releasing Insulin like growth factor (IGF-1), which causes glucose uptake and has protein anabolic effects. IGF1 exerts its effects in unbound state however during surgery, IGF binding protein (IGFBP-1) level increases and remains elevated after the operation while, IGF1 level stays unchanged or fall, resulting in decreased level of unbound IGF-1 due to reduced IGF-1/IGFBP-1 ratio. Generally Insulin reduces the production of IGFBP-1 whereas glucagon stimulates the production of this binding protein in the liver, while during surgery because of decreased insulin sensitivity the level of IGFBP-1 increases leading to reduced effect of growth hormone. All these effects lead to hyperglycemia, increased lipolysis and amino acids break down, depletion of muscle mass and net nitrogen loss. These changes contribute to increased infectious complications, morbidity, mortality and increased length of hospital stay. ${ }^{1,3}$
Volume 6 Issue 2 - 2018

\author{
Jai P Singh \\ Department of Surgery, Oswego Hospital, USA
}

Correspondence: Jai P Singh, General Surgeon, Oswego Hospital, Assistant Clinical Professor, SUNY Upstate Medical University, I I 0 W 7th Street, Oswego, New York, USA, Tel 3153 4267 7I, Fax 3 I 534227 83, Email drjp04@gmail.com

Received: April 01, 2018 | Published: April 17, 2018

\section{Effects of preoperative carbohydrate drink on metabolic state}

Preoperative carbohydrate loading decreases the insulin resistance by up to $50 \%$ and improves metabolic functions. ${ }^{3} 50 \mathrm{~g}$ oral carbohydrate drink before surgery increases insulin level to the level seen after a standard meal. ${ }^{4}$ This carbohydrate feeding induced elevation of insulin prior to or during the operation has consistently been associated with improved insulin sensitivity postoperatively. ${ }^{1}$ Increased insulin level reduces expression of PDK in skeletal muscles, thereby enabling PDC activity and carbohydrate oxidation. ${ }^{3}$ This reduction of insulin resistance by preoperative carbohydrate feeding also prevents increase in FFA level resulting decreased expression of PDK. ${ }^{3}$ Increased insulin level due to preoperative carbohydrate loading increases the insulin: glucagon ratio, which leads to decreased breakdown of body glycogen and amino acids, decreased glucose synthesis from fat or protein, and decreased fat oxidation, thus preserving body protein and fat stores. Additionally increased insulin: glucagon ratio causes increase in glycogen synthesis, lipogenesis and glycolysis. ${ }^{5}$ Attenuation of postsurgical catabolic state after preoperative carbohydrate loading occurs due to increased level of insulin like growth factor type 1 (IGF-1), which leads to improved preservation of lean body mass. ${ }^{6} 800 \mathrm{ml}(12.5 \%)$ oral carbohydrate drink $(100 \mathrm{gm})$ on the evening before the surgery and another $400 \mathrm{ml}$ $(50 \mathrm{gm})$ around $2-3 \mathrm{~h}$ before anesthesia has been found to be equivalent to intravenous dextrose infusion in reducing postsurgical catabolic state by decreasing postoperative insulin resistance. Additionally, oral drinks have the benefits of being non-invasive and convenient to the patients. ${ }^{6}$ Study by Noblett et al showed that patients who were treated with preoperative carbohydrate feeding had significantly less time to pass first flatus and bowel movement as well as decreased length of hospital stay compared to fasted group. ${ }^{2}$ Another study by Hausel et al. ${ }^{6}$ has shown that patients who were treated with preoperative carbohydrate loading had significantly decreased thirst and hunger and were less anxious compared to the patients who were fasted or had plain water. ${ }^{4}$ Moreover patients in the carbohydrate group were less hungry and less thirsty even before the morning drink, which could be explained by the continuing effect of carbohydrate loading (100gm) on the evening before the surgery. Additionally, evening carbohydrate 
loading increases glucose reserves and therefore carbohydrate treatment should be initiated on the evening before the surgery. ${ }^{4}$ Intake of $400 \mathrm{ml}$ carbohydrate drink 2-3 hours before the anesthesia does not increase the acidity or volume of stomach contents and therefore does not increase the risk of aspiration pneumonia. ${ }^{4}$

\section{Conclusion}

$800 \mathrm{ml}(12.5 \%)$ iso-osmolar or hypo-osmolar carbohydrate drink $(100 \mathrm{gm})$ on the evening of surgery and another $400 \mathrm{ml}(50 \mathrm{gm})$ around 2-3hours before the anesthesia has been proven to be safe without increasing aspiration risk and therefore should be considered before major surgical procedures except in emergency and in patients with delayed gastric emptying. This strategy helps in attenuating postoperative catabolic state and hyperglycemia and prevents depletion of muscle mass. Additionally it reduces thirst, hunger, anxiety and risk of dehydration.

\section{Acknowledgement}

None.

\section{Conflict of interest}

Author declared that there is no conflict of interest.

\section{References}

1. Thorell A, Nygren J, Ljungqvist O. Insulin resistance: a marker of surgical stress. Curr Opin Clin Nutr Metab Care. 1999;2(1):69-78.

2. Noblett SE, Watson DS, Huong H, et al. Preoperative oral carbohydrate loading in colorectal surgery: a randomized controlled trial. Colorectal Dis. 2006;8(7):563-569.

3. Awad S, Constantin Teodosiu D, Constantin D, et al. Cellular mechanisms underlying the protective effects of preoperative feeding: a randomized study investigating muscle and liver glycogen content, mitochondrial function, gene and protein expression. Ann Surg. 2010;252(2):247-253.

4. Hausel J, Nygren J, Lagerkranser M, et al. A carbohydrate rich drink reduces preoperative discomfort in elective surgery patients. Anesth Analg. 2001;93(5):1344-1350.

5. Awad S, Fearon KD, MacDonald IA, et al. A randomized cross over study of the metabolic and hormonal responses following two preoperative conditioning drinks. Nutrition. 2011;27(9):938-942.

6. Yuill KA, Richardson RA, Davidson HI, et al. The administration of an oral carbohydrate containing fluid prior to major elective upper gastrointestinal surgery preserves skeletal muscle mass postoperatively a randomized clinical trial. Clin Nutr. 2005;24(1):32-37. 